Mappemonde

Revue trimestrielle sur l'image géographique et les

formes du territoire

$125 \mid 2019$

Varia

\title{
Modélisation spatiale du récit littéraire complexe de Kateb Yacine
}

Spatial modeling of Kateb Yacine's complex literary narrative

Modelización espacial de la compleja narrativa literaria de Kateb Yacine

Juliette Morel

\section{(2) OpenEdition}

Journals

Édition électronique

URL : http://journals.openedition.org/mappemonde/766

DOI : 10.4000/mappemonde.766

ISSN : 1769-7298

Éditeur

UMR ESPACE

Référence électronique

Juliette Morel, « Modélisation spatiale du récit littéraire complexe de Kateb Yacine », Mappemonde [En ligne], 125 | 2019, mis en ligne le 01 janvier 2019, consulté le 20 juin 2019. URL : http://

journals.openedition.org/mappemonde/766 ; DOI : 10.4000/mappemonde.766

Ce document a été généré automatiquement le 20 juin 2019.

\section{cc) (†) (2)}

La revue Mappemonde est mise à disposition selon les termes de la Licence Creative Commons Attribution - Pas d'Utilisation Commerciale - Partage dans les Mêmes Conditions 4.0 International. 


\title{
Modélisation spatiale du récit littéraire complexe de Kateb Yacine
}

\author{
Spatial modeling of Kateb Yacine's complex literary narrative \\ Modelización espacial de la compleja narrativa literaria de Kateb Yacine
}

Juliette Morel

« Jules était à Berlin et au chapitre suivant, une semaine après dans le récit, nous le montrons à

Strasbourg, le trajet, le voyage étant passé sous silence. Le personnage, lui, a bien été obligé de traverser tous les intermédiaires, nous les sautons. Nous habitons l'espace romanesque autrement que lui, mais cette "habitation" va poser des problèmes similaires. Entre la composition romanesque et l'organisation réelle de l'espace habité sous toutes ses formes : chambre, ville, ou la terre entière, il y a d'étroites relations, puisqu'il s'agit de traiter,

d'organiser les parcours, ici réel, là fictif, d'une chambre à l'autre, d'un quartier, d'une ville à

l'autre. ", Michel Butor, « Philosophie de l'ameublement ", Essais sur le roman, 1992, p. 70-71.

Kateb Yacine, écrivain algérien né en 1929 et mort en 1989, est l'auteur d'une œuvre qui se compose de deux périodes : une première série d'œuvres écrites en langue française avant 1968, dont les principales sont Nedjma, roman publié en 1956, Le Cercle des représailles, recueil théâtral publié en 1959, et Le Polygone étoilé, œuvre au genre hybride publiée en 1966 ; et une deuxième série de pièces satiriques populaires, élaborées et jouées de 1970 à la fin des années 1980 en arabe dialectal en Algérie. La première partie de cette œuvre, rassemblée par la critique sous le nom de «Cycle de Nedjma " (Nagget Khadda, 2002, p. 141), constitue le socle solide sur lequel toute l'œuvre katébienne est bâtie, à la fois foisonnante et hétérogène, mais cohérente et unifiée. C'est sur celle-là que porte notre étude. Elle regroupe des textes ayant pour point commun d'avoir les mêmes 
personnages (quatre jeunes amis et une femme - Nedjma - dont ils sont tous amoureux), le même contexte géographique et historique (l'Algérie des années 1940-1960, la colonisation et la guerre d'indépendance), le même engagement politique et les mêmes thèmes principaux (l'anticolonialisme, l'identité ancestrale, l'amour, la nation). De cette manière, le Cycle de Nedjma est complexe et « tisse ensemble » (E. Morin) - c'est-à-dire met en relation en les assemblant dans une même unité - des fragments hétérogènes d'un point de vue éditorial, d'un point de vue du genre, de la tonalité, de la focalisation, du continuum spatio-temporel, etc.

\section{Une œuvre littéraire à la spatialité complexe}

2 L'œuvre de Kateb Yacine est spatiale à deux égards. D'une part, c'est une œuvre qui, bien qu'elle soit fictionnelle, s'ancre dans le contexte géographique et historique de l'Algérie des années 40-60 et aborde des thèmes géopolitiques réels : la colonisation, la lutte pour l'indépendance, la nation, l'identité, le territoire. Cette œuvre - comme toute œuvre narrative - parle d'espace et de géographie en tant qu'elle est une construction et une expression d'un rapport entre l'homme (le narrateur, le personnage, l'auteur, la communauté) et l'étendue terrestre sur et dans laquelle il vit (Di Meo, 2001; Berque, 1996 ; Levy et Lussault, 2003). Il s'agit de la représentation d'un espace externe à l'œuvre, auquel celle-ci fait référence. L'espace géographique, selon l'emploi que nous ferons de ce terme, a donc une dimension référentielle et réaliste importante; bien que l'espaceréférence soit toujours inclus et transformé par la représentation littéraire - par le point de vue qu'elle adopte, par ce qu'elle en dit et par la forme qu'elle adopte. D'autre part, les récits de Kateb Yacine sont marqués par une narration complexe à la chronologie et à la géographie éclatée ; et sa création poétique est marquée par une construction en réseau et des réécritures cycliques. Kateb Yacine compare la structure narrative et la construction poétique de son œuvre à un espace dont la structure est géométrique, circulaire, cyclique, ou en forme de polygone étoilé - le polygone étoilé décrivant les « plans bouleversés » de l'œuvre (Kateb Yacine, 1966, p. 10, 11, 13, 96, 131 ; Kateb Yacine, 1994, p. 41-42 ; Gontard, 1985 ; Arnaud, 1986 ; Bonn, 2000 ; Morel, 2014, 2016). Cette caractérisation métalittéraire s'apparente à certains égards à « l'espace littéraire » que développe la théorie littéraire depuis le milieu $\mathrm{du} \mathrm{xx}^{\mathrm{e}}$ siècle, en tant qu'il désigne les phénomènes de spatialité ou d'espacement $d u$ texte provoqués par des procédés spécifiquement littéraires. L'espace littéraire peut en ce sens renvoyer aux jeux sur la typographie, aux juxtapositions de genres, de tonalités, de focalisations différentes qui donnent de la granulosité au texte (Butor, 1992), aux phénomènes d'intertextualité ou d'hypertextualité qui lui confèrent complexité et "épaisseur " (Genette, 1982), aux figures qui creusent des «espaces» ou «étendues» de sens (Genette, 1966), ou encore aux réseaux sémiotiques formés par les liens transversaux reliant et espaçant les nœuds du texte (Blanchot, 1955; Guere, 1990; Garnier et Zoberman, 2006). Nous incluons également dans la notion d'espace littéraire les effets de distorsion provoqués par le récit entre la diégèse - c'est-à-dire l'histoire racontée, dimension descriptible particulièrement par ses paramètres spatio-temporels - et la narration - c'est-à-dire la manière dont cette histoire est racontée, dimension descriptible par les paramètres matériels du déroulement du texte (Genette, 1972). C'est selon ces acceptions, qui pour la plupart considèrent le texte dans sa dimension matérielle, que nous entendons le terme d'espace littéraire et que nous déclarons que l'œuvre de Kateb Yacine est un espace. 
3 Nous entendons décloisonner ces deux domaines spatiaux - espace géographique et espace littéraire - pour rechercher ce qu'il y a de commun entre eux, avec en vue l'horizon idéal « d'une théorie unitaire de l'espace » (Lefebvre, 1974) et la possibilité de construire des méthodes et des outils qui les mettent en relation. Les deux sphères spatiales décrites (géographique et littéraire) ne sont en fait pas indépendantes chez Kateb Yacine, mais elles sont au contraire co-construites. Le lien entre les deux dimensions spatiales à priori de natures différentes est notamment établi dans le texte de Kateb Yacine par des métaphores filées que nous avons déjà commencé à évoquer: les métaphores géométriques. Les figures géométriques récurrentes apparaissent toujours dans le texte de Kateb Yacine premièrement comme des métaphores de l'espace, de phénomènes sociaux-politiques référentiels ou d'éléments biographiques réels, puis évoluent vers des significations conceptuelles et métalittéraires, jusqu'à établir les plans métaphoriques des structures narratives et/ou poétiques à différentes échelles. Elles s'ancrent en effet dans un premier temps dans une réalité contextuelle et biographique externe : la cousine Nedjma motive la métaphore de l'étoile (Nedjma signifiant étoile en arabe); l'éternel retour et l'angoisse temporelle partagés par de nombreux auteurs contemporains motivent la métaphore du cercle (Kateb Yacine, 1994: 41-42; Kateb Yacine, 1966: 39); la situation géopolitique de l'Algérie et les conventions cartographiques coloniales motivent la métaphore du polygone (Kateb Yacine, 1959 : 126). Dans un deuxième temps, par une dérive de sens fondée sur les propriétés géométriques formelles de chacune de ces figures, celles-ci participent activement aux processus de construction du système poétique katébien et de la structure narrative. Ainsi les trois figures de l'étoile, du cercle et du polygone participent à la cohérence verticale, pourraiton dire, de l'œuvre de Kateb Yacine : depuis son ancrage dans le réel et son engagement politique jusqu'aux éléments les plus métatexuels et autoréférentiels. En outre, par leurs répétitions et leurs échos, ces figures participent aux réseaux horizontaux qui structurent le système katébien. Elles peuvent être ainsi considérées comme des éléments combinatoires, à l'échelle du Cycle de Nedjma; le résultat de leur combinaison forme un polygone étoilé, de l'aveu de l'auteur lui-même (Kateb Yacine, 1994, p. 41). Les figures géométriques font ainsi le lien entre la prise sur le monde de l'œuvre, que Kateb Yacine affirme régulièrement dans ses commentaires, et l'autonomie très forte du texte dont la structure et les thématiques fonctionnent selon des réseaux internes ; autrement dit, elles lient l'espace géographique référentiel et l'espace littéraire.

4 La notion d'espace littéraire, la caractérisation géométrique endogène de l'œuvre de Kateb Yacine et la relation entre espace littéraire et espace géographique qui y est établie permettent d'aborder certains phénomènes poétiques et narratifs comme s'ils se déployaient dans l'espace. Il est ainsi possible de les mettre directement en relation avec l'espace géographique qu'ils représentent et d'utiliser les mêmes concepts, les mêmes méthodes et les mêmes outils pour les étudier. De la même manière que le poète modélise symboliquement le récit grâce au polygone étoilé, nous proposons d'adopter une démarche de modélisation spatiale pour comprendre le fonctionnement spatial de l'œuvre. L'objectif de cette modélisation est de formaliser concrètement - et non plus seulement théoriquement comme nous l'avons fait jusqu'ici - les relations entre les différentes spatialités en jeu dans une œuvre littéraire comme celle de Kateb Yacine. Dans cette perspective, nous entendons modéliser le récit katébien en suivant les principes des systèmes d'information géographique et des systèmes d'information spatio-temporelle parce que le récit est le résultat d'une manipulation du temps et de l'espace par la 
narration - pour décrire dans un même système d'information la structure du récit et la géographie de l'œuvre. Nous pourrons ainsi explorer précisément leur interrelation, leur co-construction, autrement dit l'influence que le récit a sur la géographie de l'œuvre et inversement l'influence de l'espace géographique référentiel sur le récit, question qui anime in fine cette recherche. La suite de cet article a pour objectif de décrire les choix méthodologiques effectués pour construire ce système d'information spatio-narratif.

\section{Essai de formalisation du récit de Kateb Yacine dans un Système d'informations spatio-narratif}

5 L'objet central de notre modélisation est le récit, c'est-à-dire le rapport entre l'histoire qui est racontée - que nous appellerons la "diégèse » - et la manière dont elle est racontée - que nous appellerons la «narration» (Genette, 1972: 15). Il s'agira de découper le récit en objets narratifs et de les localiser les uns par rapport aux autres pour en mesurer la proximité (la cohérence), l'éloignement (l'éclatement) et les motifs ainsi formés (répétitions, flash-back, flash-foward, ellipses, etc.). Se posent à partir de là deux questions de méthode: premièrement celle de la définition des objets narratifs et deuxièmement celle de leur localisation, c'est-à-dire la définition des dimensions du système d'information. Nous précisons que l'expérience de modélisation spatio-narrative décrite dans la partie qui suit a été appliquée au seul roman Nedjma, considéré comme un extrait représentatif choisi par souci pragmatique d'applicabilité, mais que ses principes peuvent - et devraient idéalement - s'appliquer au reste du Cycle de Nedjma.

\section{Comment délimiter les objets unitaires au centre du modèle ?}

6 En linguistique, lexicologie numérique ou statistiques textuelles, on a tendance à faire correspondre les unités étudiées à des unités formelles objectives : le phonème, le mot, la phrase, le paragraphe, le chapitre, la scène ou l'acte de théâtre, dépendant de l'objet et de l'objectif de l'analyse (Hoover, 2007, p. 3). Dans notre cas, il s'agit d'étudier le récit ; or le récit peut se découper en segments narratifs selon les principes de la narratologie. La définition de ces segments repose sur des critères non seulement formels, mais également diégétiques. La difficulté est de faire coïncider de manière homogène le segment textuel avec l'unité de sens narratologique, à une échelle intermédiaire entre le mot et le chapitre. Cette difficulté est d'autant plus grande dans le cas d'une prose issue d'expérimentations formelles comme celle de Kateb Yacine, où le foisonnement, l'éclectisme et l'irrégularité des modes, des voix et du temps narratifs déstructurent les découpages matériels du texte à première vue réguliers et symétriques (Nedjma est composée de 6 chapitres, chacun divisé en 12 ou $2 \times 12$ sous-chapitres). Une telle approche introduit donc forcément un biais d'interprétation dans la méthode, nécessaire pour garantir le respect de l'objet d'étude littéraire, mais dont il faut avoir conscience pour limiter la partialité qu'il peut provoquer.

7 Pour affronter cette difficulté, nous faisons reposer notre méthodologie sur des principes narratologiques développés par Gérard Genette et Roland Barthes (dans la droite ligne d'une conception matérielle et structuraliste du texte). Genette propose de fonder son analyse de l'achronie narrative sur des « segments narratifs » qu'il délimite en suivant « la présence d'une rupture temporelle et/ou spatiale importante » dans le récit (Genette, 1972, p. 83-84). La rupture spatio-temporelle est le critère sur lequel se fonde notre 
découpage exhaustif du texte en objets narratifs unitaires. Néanmoins, celui-ci manque de précision quant à l'échelle des objets narratifs : tout dépend de ce qu'on entend par « ruptures importantes ». Pour répondre à ce problème et rendre compte du fait que des segments narratifs de grandeurs différentes se côtoient, s'emboîtent, s'enchainent, il a été nécessaire de définir plusieurs échelles d'objets narratifs. Nous faisons reposer ce principe sur une notion narratologique de Barthes: celle des différents «niveaux » ou "étages» de récit agencés hiérarchiquement. Le critique explique en effet que "si chacun a ses propres unités et ses propres corrélations, obligeant pour chacun d'eux à une description indépendante, aucun niveau ne peut à lui seul produire du sens" (Barthes, 1966, p. 5). Selon ce principe, nous définissons trois niveaux d'objets narratifs. Premièrement, nous appliquons des délimitations à une très grande échelle et de manière absolument systématique : à chaque fois que le récit change de temps et/ou d'espace, une nouvelle unité narrative est définie. On définira par exemple deux objets narratifs pour différencier d'une part un passage de Nedjma se déroulant dans la maison où vit Nedjma, située à Bône ${ }^{1}$, et d'autre part un passage se déroulant juste après dans une autre partie de la ville de Bône. C'est ainsi que sont définis objets narratifs les plus fins (de niveau 1), qui font en moyenne un peu moins d'une page dans Nedjma. Deuxièmement, des objets narratifs d'échelle intermédiaire (de niveau 2) sont définis lorsque des ruptures plus importantes de temps ou de lieu sont constatées : par exemple lorsqu'on passe d'un récit se déroulant dans la ville de Bône à un récit se déroulant dans la ville de Constantine. Ces récits font en moyenne 2 pages dans Nedjma; ils correspondent à peu près à l'échelle des sous-chapitres (même s'ils n'en épousent pas exactement les limites). Troisièmement, de grandes unités narratives (de niveau 3) sont définies pour délimiter les ruptures spatiotemporelles radicales : elles signalent des ruptures achroniques (analepses, prolepses ou ellipses) ou des sauts spatiaux importants en termes de distance et passés sous silence (bien qu'il existe aussi des achronies et des sauts spatiaux au niveau narratif 1 et 2, les délimitations du niveau 3 en relèvent systématiquement). Ces unités de niveau 3 sont au nombre de 15 dans le roman et font en moyenne 16,3 pages. 
Figure 1. Représentation graphique des petits objets narratifs selon le critère géographique

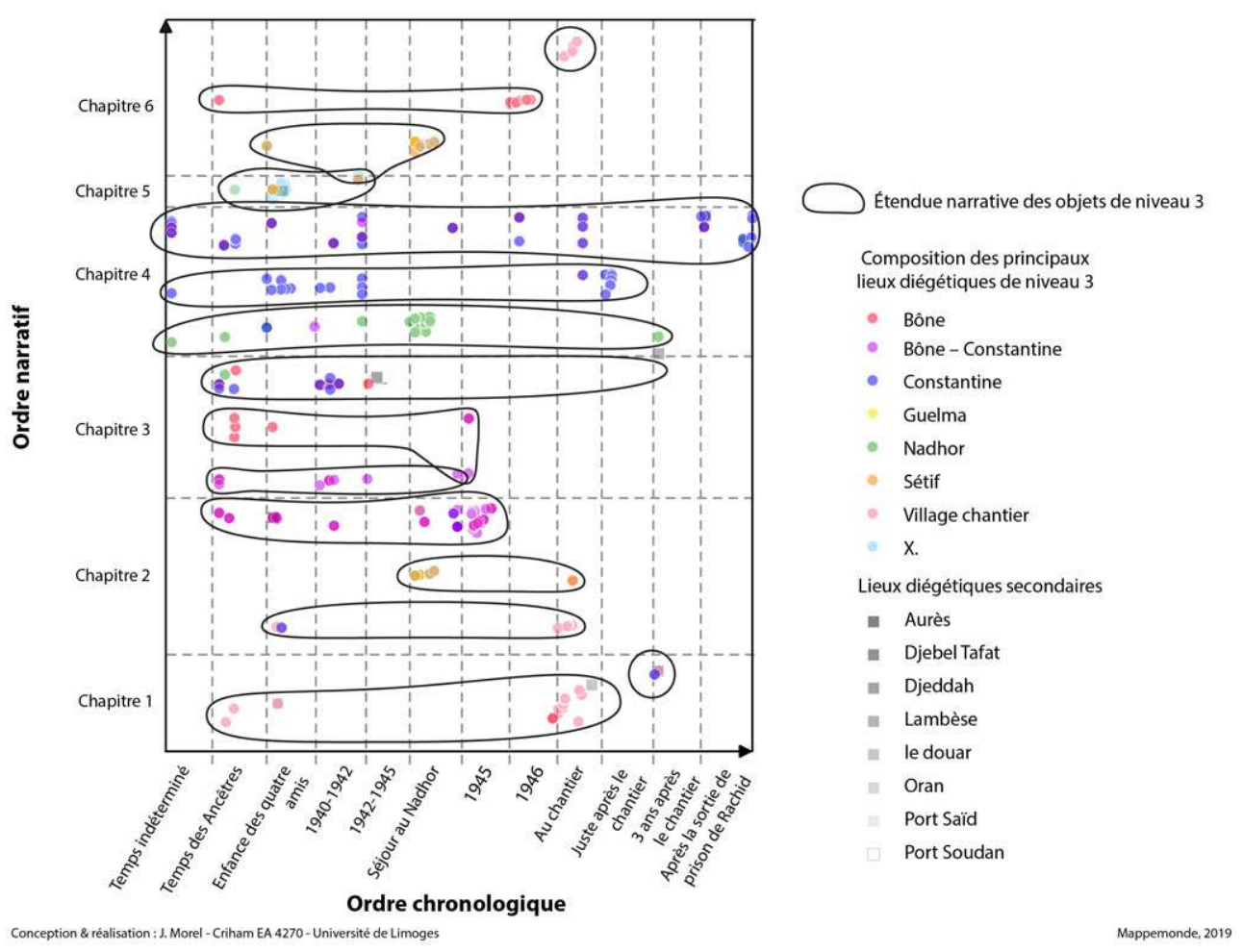

Conception \& réalisation : J. Morel- CRIHAM, Université de Limoges

8 Le graphique ci-dessus (figure 1) représente les localisations narratives des plus petits objets narratifs (de niveau 1). Il indique l'espace géographique dans lequel chacun d'eux se déroule (un des critères de délimitation de ces objets) et la manière dont ils sont rassemblés pour former les plus grands objets narratifs de niveau 3. L'observation de ce graphique donne une première information sur le rapport entre espace et récit chez Kateb Yacine : l'espace - bien qu'il ne soit jamais le seul critère - semble intervenir davantage que le temps dans la délimitation des grands objets narratifs. En effet, sauf exception, les grandes unités ont une large étendue sur l'axe de la chronologie, par contre on observe la prééminence d'un ou de deux lieux dans chacune, donc une cohérence géographique.

Dans la base de données spatio-temporelles, l'appartenance des objets narratifs aux différents niveaux sera intégrée, mais ceux-ci seront formalisés et stockés de la même manière, c'est-à-dire comme des objets de grandeurs différentes (mesuré en nombre de mots), mais de même nature. Ceci est fondamental pour modéliser un certain jeu sur les échelles, propre, selon nous, à l'œuvre de Kateb Yacine qui non seulement emboîte, mais aussi confond, échange et multiplie les différentes échelles narratives dans son polygone étoilé. Un même épisode peut être raconté plusieurs fois avec des longueurs variables, les sous-chapitres peuvent avoir des longueurs très différentes ${ }^{2}$ et une importance diégétique inversement proportionnelle à leur longueur ${ }^{3}$. Si les unités d'échelles différentes s'emboîtent, les grandes unités de niveau 3 n'équivalent pas pour autant à la somme des unités de niveaux 2 ou 1 qui les composent; l'ensemble peut connoter, impliquer et signifier davantage que la somme de ses parties. L'exploration des relations entre les objets narratifs de même niveau (horizontales) et de niveaux différents 
(verticales) sera à cet égard très instructive. Dans cet objectif, passons maintenant aux critères de localisation des objets narratifs les uns par rapport aux autres au sein du récit.

\section{Les dimensions du système d'information spatio-narratif}

10 En appliquant à la lettre les principes de la narratologie de Gérard Genette, localiser les objets narratifs dans le récit consiste à les situer premièrement dans l'espace de la diégèse (où se situe tel épisode dans l'espace géographique référentiel, réel ou non?), deuxièmement dans la chronologie de la diégèse (à quel moment se situe tel épisode dans le déroulement temporel de l'histoire?) et troisièmement dans le déroulement de la narration (à quel endroit du texte se trouve le récit de cet épisode ?). Nous modélisons le système narratif ainsi décrit en nous inspirant des systèmes d'information spatiotemporels utilisés en histoire, géographie ou archéologie modélisant l'information à fois spatiale, temporelle et thématique. Certaines de ces modélisations insistent sur le fait de considérer et de stocker les différentes dimensions du système - spatiale, temporelle et thématique - comme des informations autonomes et externes aux objets modélisés, et non comme des modalités de ceux-ci, afin de pouvoir interroger le modèle à travers n'importe laquelle de ces dimensions - et non seulement à travers les objets centraux et ainsi garantir la flexibilité des requêtes (Rodier, Saligny, 2010 ; Plumejeaud, Cristofoli, Motte, 2015). Dans le cas de la modélisation spatio-narrative du récit de Kateb Yacine les dimensions à modéliser sont les suivantes : la dimension géographique, mesurée par la latitude notée $\mathrm{x}$ et la longitude notée y (que l'implantation soit ponctuelle, linéaire ou surfacique), la dimension chronologique diégétique mesurée par une datation historique, notée $t$, et la dimension matérielle du texte qui traduit la narration, mesurée au rang du mot et notée $m$ (le mot pourra au besoin être agrégé à d'autres niveaux de texte parfois plus signifiants : phrase, page, sous-chapitre, chapitre, ouvrage). Les objets narratifs sont donc localisés dans un système d'information spatio-narratif (SISN) en quatre dimensions : x.y.t.m. Une fois ces dimensions posées, il est nécessaire de détailler la manière dont nous allons les mesurer.

\section{La localisation géographique de l'objet narratif (dimensions $x$ et y du SISN)}

Le premier aspect - et le plus important concernant la question de la spatialité du récit posée dans cet article - concerne la manière dont les objets narratifs seront géographiquement localisés (dimension 1 et 2 du SISN), sachant que la géographie d'un roman peut se mesurer de diverses manières. L'importance d'un lieu dans un roman peut être définie par la manière dont il est convoqué : les lieux cités directement et sans ambiguïté par leurs toponymes dans le texte n'ont pas le même statut que les lieux auxquels il est fait référence indirectement et qui nécessitent pour certains l'interprétation du lecteur (gentilé, dénominations génériques du type " mon pays », « la rivière ", périphrases du type "le pays dont nous venons", adverbes spatiaux du type « ici », « là-bas", localisation relative du type " de l'autre côté du détroit », etc.). Parmi les 585 citations de lieux dans Nedjma, 288 sont des citations directes par toponyme, et 298 sont des citations indirectes. De même, les lieux qui ne sont que cités n'ont pas la même fonction, ni par conséquent le même statut, que les lieux dans lesquels se déroule l'action, les lieux diégétiques (voir la figure 2 et la figure 3). Nous avons intégré dans le modèle tous ces types d'espaces, leur modalité de présence dans le texte et leur fonction. Il est d'autant plus nécessaire de signifier ces distinctions dans la référentialité spatiale 
du roman que les centres de gravité géographiques qui en résultent sont très différents dans Nedjma (comme en témoigne la comparaison des figures ci-dessous).

Figure 2. Lieux cités dans Nedjma

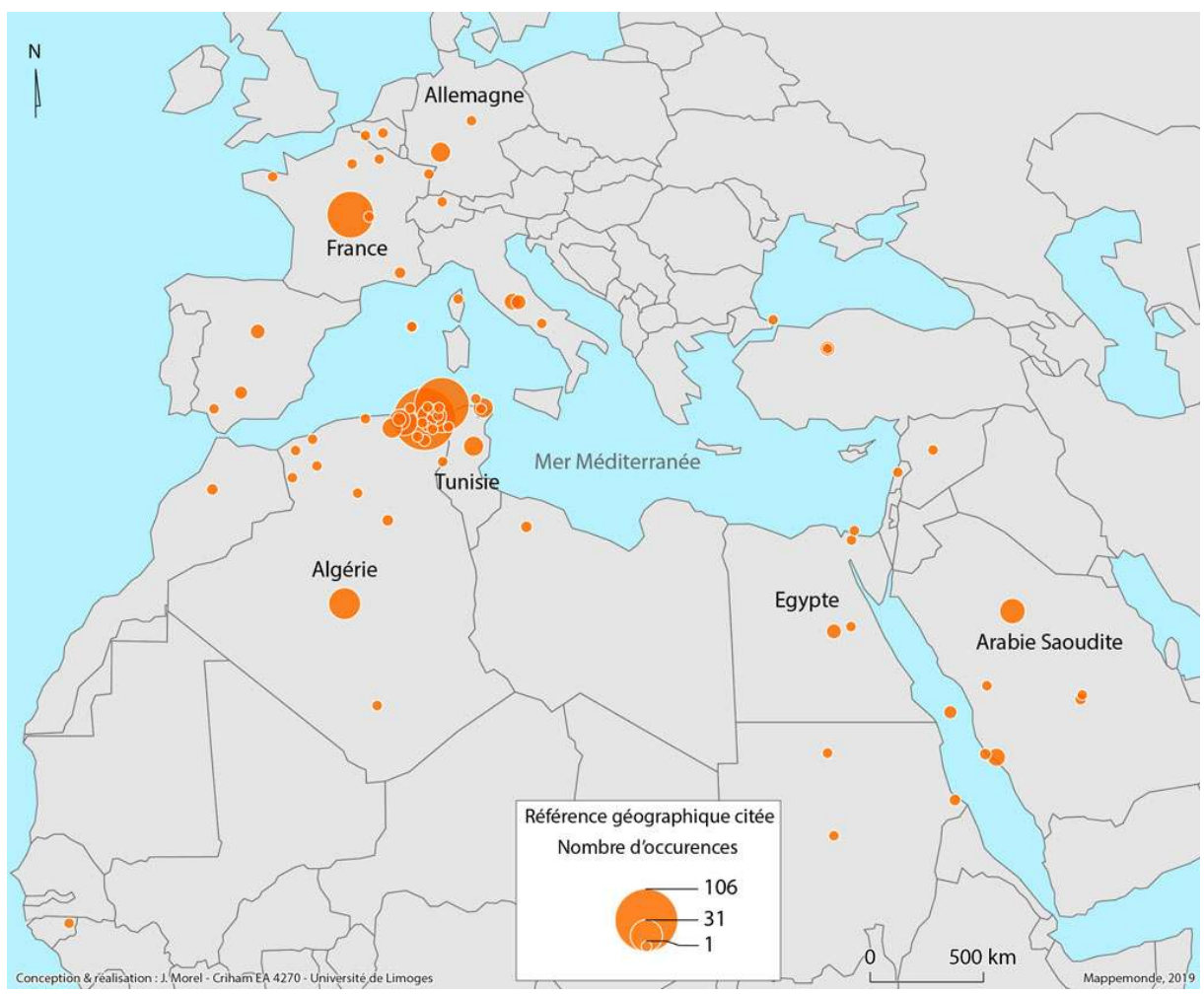

Conception \& réalisation : J. Morel- CRIHAM, Université de Limoges 
Figure 3. Lieux de la diégèse de Nedjma

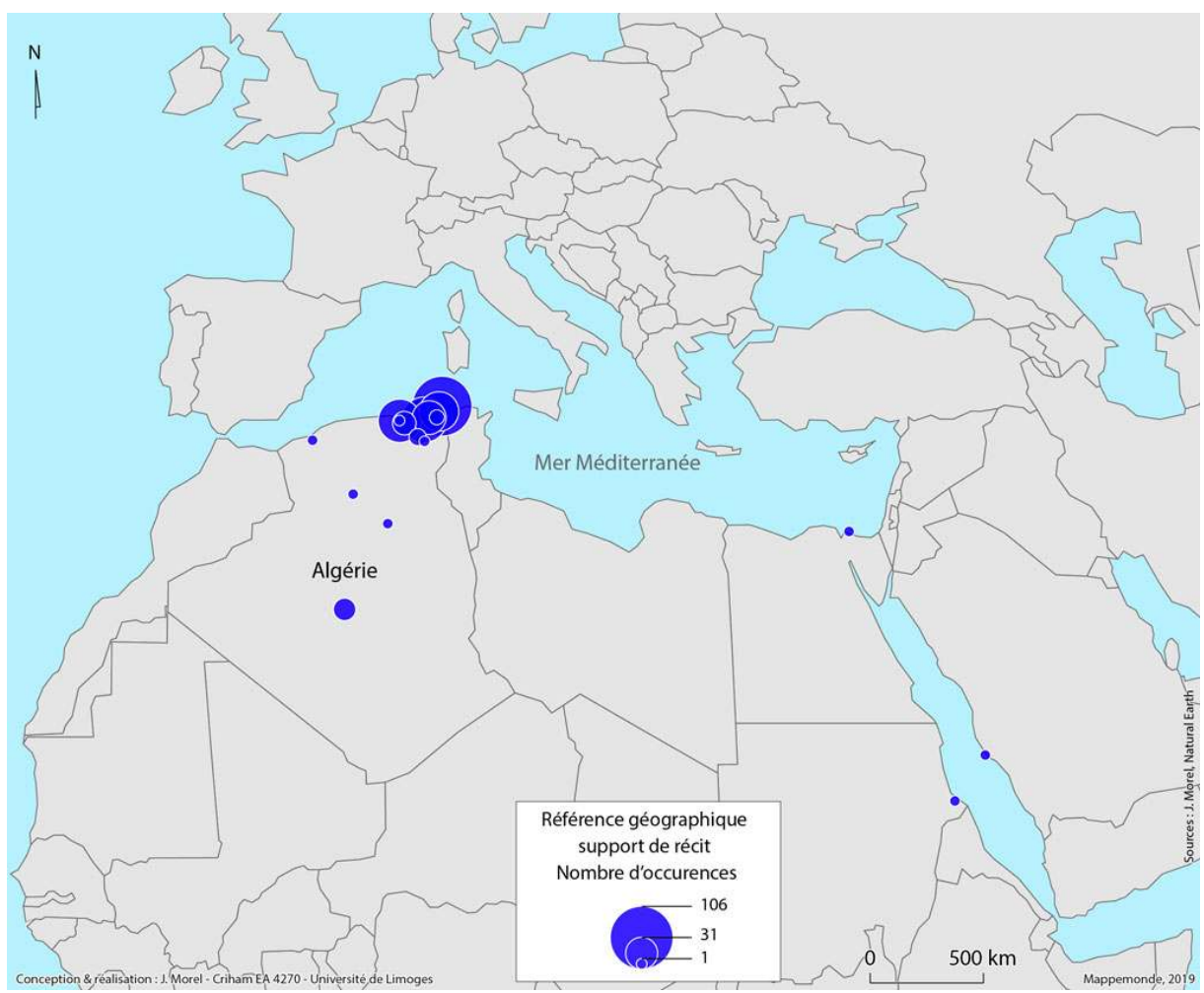

Conception \& réalisation : J. Morel- CRIHAM, Université de Limoges

Une fois ces distinctions précisées, concentrons-nous sur la formalisation des lieux diégétiques qui sont ceux qui entrent en jeu dans la définition géographique de nos objets narratifs. La fixation de ces lieux diégétiques, puisqu'ils ne sont pas forcément liés à une citation claire et directe dans le texte, pose les problèmes de la délimitation, de l'uniformisation (conséquent au caractère hétérogène de l'information) et de l'exhaustivité des données. Délimiter les données spatiales issues d'œuvres narratives sur le seul critère géographique pourrait impliquer les dilemmes suivants : le passage d'une rue à une autre rue adjacente au sein d'un même ensemble narratif, par exemple, doit-il être considéré comme un changement de lieu géographique? Que faire alors d'une description suivant les déambulations d'un personnage qui traverserait plusieurs lieux ? Doit-on les différencier ou considérer ce choix narratif comme la représentation d'une pratique spatiale continue, reliant des lieux pour créer un nouvel espace cohérent à une échelle inférieure? Se focaliser sur les changements de lieu reviendrait alors à nier la capacité de la littérature (et de la narration en générale) à créer de nouveaux espaces (Morel, Rouiai, 2016). Nous nous servirons de la cohérence spatio-temporelle utilisée pour délimiter les objets narratifs et décrite ci-dessus afin de résoudre cette difficulté et en déduire les unités géographiques de l'œuvre. Ainsi unité narrative et unité géographique se définissent mutuellement en dialogue, l'une participant à la définition de l'autre, et inversement, jusqu'à atteindre un état d'équilibre spatio-narratif. Le problème de la délimitation rejoint en fait la question de la précision et de l'uniformisation des données, notamment en termes d'échelles. Si l'on extrait une information à l'échelle de la rue pour certains objets narratifs, est-on assuré que ce degré de précision scalaire sera retrouvé tout au long du récit? Et si ce n'est pas le cas, peut-on comparer une donnée extraite à 
l'échelle de la rue avec une donnée extraite à l'échelle de l'unité urbaine, voire du pays ? Pour répondre à ces questions, nous avons associé aux informations de lieu une caractérisation d'échelle. Sont définies quatre échelles spatiales différentes (échelle 1 infra-urbaine, échelle 2 urbaine, échelle 3 régionale, échelle 4 nationale ou continentale), de la même manière que nous avions défini trois échelles narratives. Cela ne signifie pas qu'aux objets narratifs les plus grands seront forcément associées des unités géographiques de petite échelle (pays, continent) et inversement pour les objets narratifs les plus petits. L'association d'un grand objet narratif à une petite unité géographique est une information en soi que nous ne devons pas invisibiliser par une formalisation excessive des données: ce fait indique un recentrement géographique qui peut être révélateur d'une tension narrative. Par exemple dans Nedjma, parmi les trois grands objets narratifs de niveau 3 composant le chapitre IV, deux sont localisées à Constantine, qui est un lieu d'échelle 2 (échelle urbaine). Cette association connote un immobilisme fort au chapitre IV autour du personnage de Rachid, ancré dans cette ville, qui apparait comme le cœur géographique et thématique du chapitre. D’autres grands objets narratifs consistent au contraire à raconter la mobilité des personnages d'un lieu à un autre; ils sont associés à des espaces d'échelle $3:$ « Est de l'Algérie » (5 grands objets sur 15) et « De Bône à la Mecque » (1 grand objet sur 15, qui correspond au récit du pèlerinage de $\mathrm{Si}$ Moktar et Rachid).

Figure 4. Graphe des liens entre les lieux de Nedjma selon l'enchaînement narratif des récits de niveau 1 (les récits les plus petits)

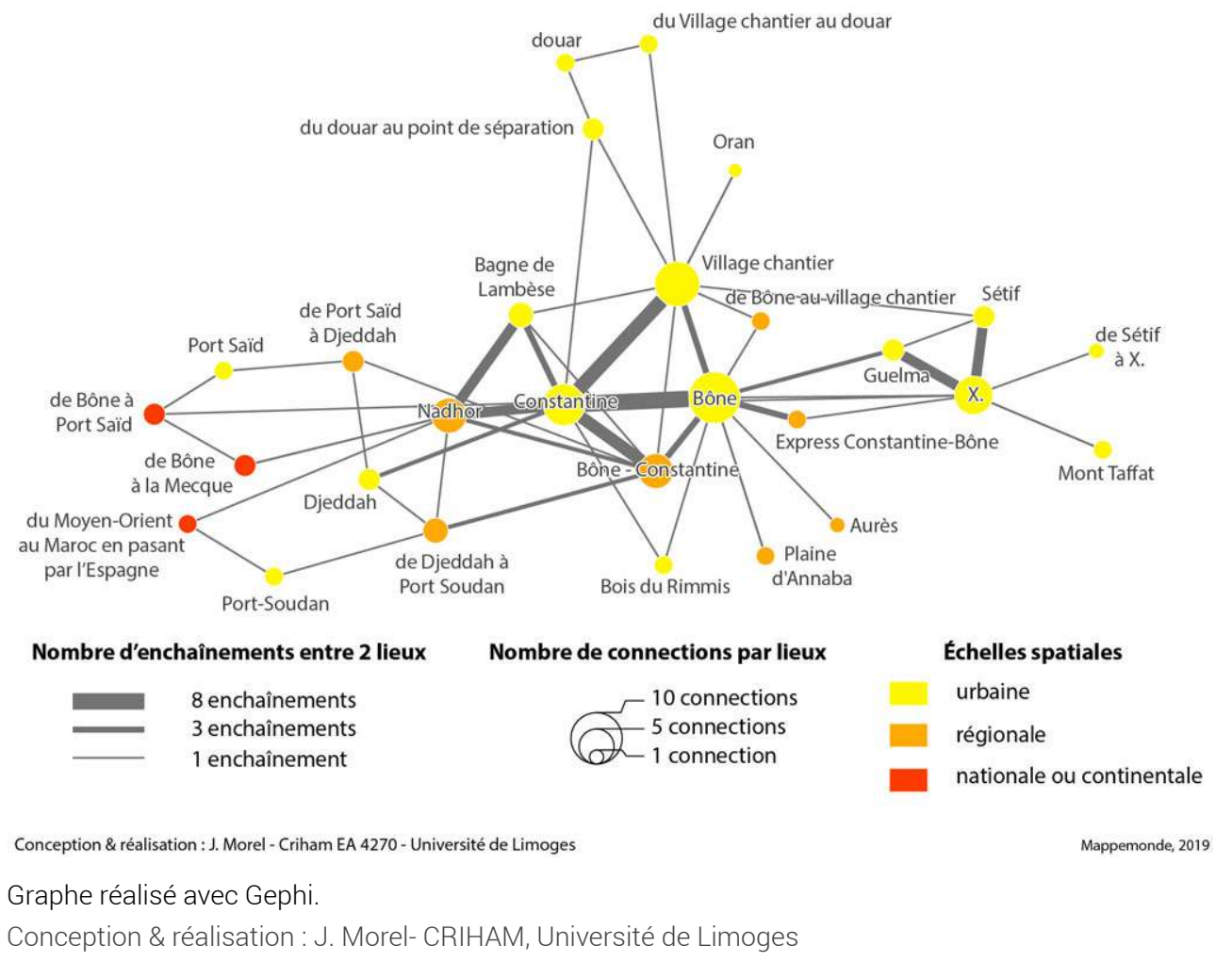

13 La figure 4 recense tous les lieux où se déroulent les petits objets narratifs de niveau 1 , agrégés au niveau minimal de l'urbain (dans un souci de synthèse, les lieux d'échelle infra-urbaine ne sont pas représentés). Considérer ces lieux d'échelles géographiques différentes de manière équivalente, parce qu'ils correspondent à une même échelle 
narrative, implique qu'il est difficile de les représenter sur la trame d'un fond de carte topographique. La topologie du graphe redessine l'espace sur la base de la connectivité narrative des lieux (les lieux les plus connectés entre eux sont les plus proches). Il est intéressant de constater que les lieux centraux dans la narration (donc centraux dans le graphe) sont traités à des échelles fines alors que les lieux périphériques en termes narratifs sont davantage considérés à des échelles inférieures (la plaine d'Annaba, « du Moyen-Orient au Maroc, en passant par l'Espagne ", etc.). Cela semble logique : les lieux où se déroule la plus grande partie de l'histoire sont plus précisément connus et décrits. Plus un espace se précise en terme diégétique et plus il est traité à grande échelle. À l'inverse, les lieux périphériques d'un point de vue diégétique sont traités à une échelle plus petite et plus généralisante.

Une dernière difficulté se pose pour la localisation géographique des objets narratifs: celle de l'ambiguïté. Comment traiter les objets narratifs dont la localisation est ambigüe ou insuffisamment précise et les intégrer à un ensemble d'autres objets dont la localisation est précise? Car la spécificité du discours spatial porté par l'œuvre d'art réside justement dans la possibilité de l'ambiguïté, dans la mise en pratique de son droit à l'omission, dans le rapport qu'elle construit entre l'implicite et l'explicite. Le problème de la localisation géographique (quelles variables $\mathrm{x}$ et $\mathrm{y}$ ?) se pose alors de nouveau: comment reporter ces lieux ambigus, tout aussi importants que les autres, sur la projection de l'espace géographique référentiel? Dans Nedjma par exemple, certaines localisations sont particulièrement problématiques : la ville de « $\mathrm{X}$. » - l'indétermination est volontairement explicite dans son toponyme - et le « village » dans lequel les quatre protagonistes vont travailler - qui ne sera jamais plus précisément désigné (figure 5).

Figure 5. L'espace diégétique de Nedjma

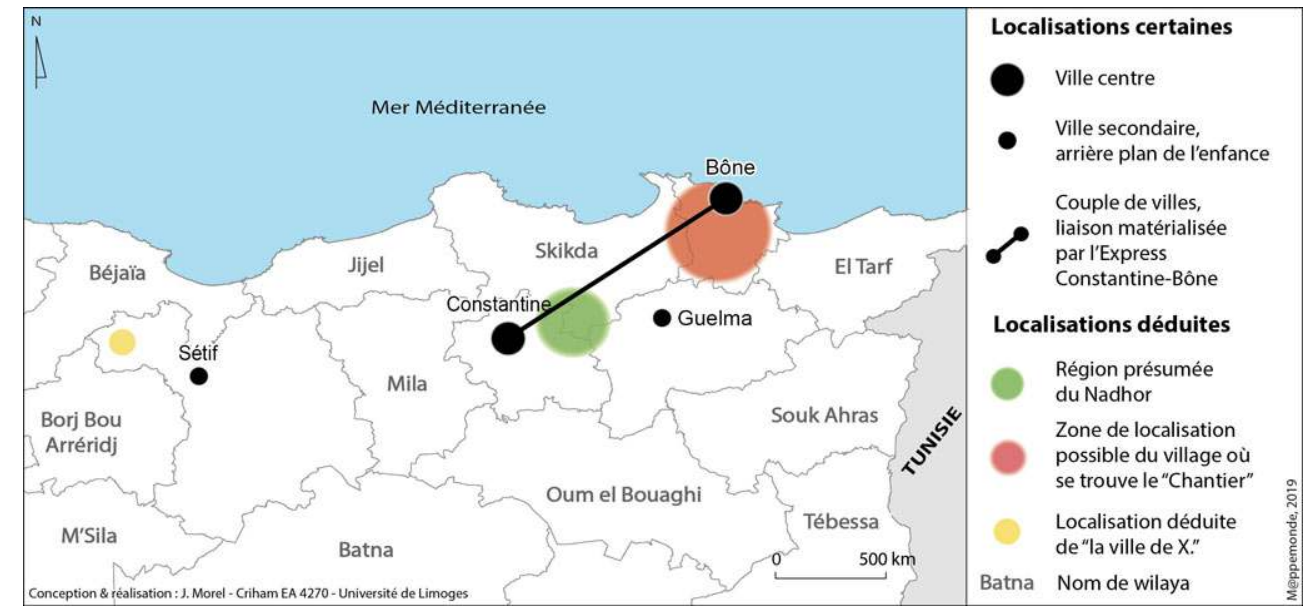

Conception \& réalisation : J. Morel- CRIHAM, Université de Limoges

Pour la ville de X., il est possible de déduire une localisation précise : elle se trouve à 50 kilomètres de Sétif (Kateb Yacine, 1956, p. 186) au pied du mont Taffat. En revanche, le village dans lequel se trouve le chantier n'est situé qu'en altitude (Kateb Yacine, 1956, p. 20). On constate seulement qu'il est assez proche de Bône pour qu'une ligne directe et quotidienne de bus le relie à la ville (Kateb Yacine, 1956, p. 11-14). Pour garantir leur prise en compte dans le modèle, nous avons choisi de valider la localisation déduite pour la ville de X. et de forcer une localisation pour le village du chantier. Néanmoins, l'objet géographique résultant est associé à un attribut sémantique signifiant son ambiguïté. Un 
autre problème peut se poser: celui de la cohabitation d'espaces réels et d'espaces imaginaires impossibles à reporter sur une carte géographique de la Terre. Ce problème ne se posant pas chez Kateb Yacine qui traite uniquement de lieux réels ${ }^{4}$, nous le laissons de côté. Restent enfin des espaces indéterminés qu'il est impossible de fixer ou de déduire. Pour ne pas tomber dans l'écueil du relativisme ou au contraire de la localisation à tout prix et quoiqu'il en coûte et pour prendre en compte ces espaces dans nos mesures, nous avons choisi d'associer ces informations à de nouvelles catégories spatiales l'ailleurs et l'indéfini.

\section{Localisations chrono-diégétique et narrative de l'objet narratif (dimensions 3 et 4 du SISN)}

Les deux autres dimensions de localisation des objets dans le système narratif concernent l'ordre chrono-diégétique et la situation du passage dans la narration. Le premier aspect pose les mêmes questions de délimitation, d'échelle et de comparaison que les localisations géographiques dans la mesure où les références historiques associées aux épisodes narratifs dans Nedjma peuvent correspondre à des pas de temps très différents des temps antiques flous à la "matinée du 8 mai 1945 »-, dans la mesure où certains épisodes ne peuvent être situés chronologiquement que relativement aux autres, voire où leur chronologie ne peut être fixée définitivement pour cause d'incohérence (c'est le cas de l'épisode du Nadhor qui revêt de ce fait un caractère irréel, imaginé). Pour modéliser la complexité et la multiscalarité temporelle de la narration, les unités temporelles que nous avons définies dans la modélisation ont donc des pas de temps historiques différents (comme les objets narratifs et les lieux avaient des échelles différentes), mais correspondent à un statut diégétique similaire. Est assigné à chacune son rang relatif dans l'ordre de la linéarité historique. La comparaison de la position des objets narratifs dans cet ordre chronologique historique linéaire et de leur position dans l'ordre de la narration - dernière dimension que nous allons aborder tout de suite - permet de rendre compte de la manipulation de la temporalité par le récit katébien. Elle révèle une temporalité pointilliste, éclatée, voire cyclique - lointaine de la conception historique linéaire. Pour finir, la quatrième dimension du système correspond à la position des objets narratifs dans l'ordre de la narration, c'est-à-dire la place qu'ils occupent dans le déroulement du texte. Cette dimension est la seule à pouvoir être mesurée de manière strictement matérielle, en indiquant le rang du premier et du dernier mot les composant, considérant le mot comme l'unité textuelle de base.

\section{Implémentation de la modélisation dans une base de données relationnelle}

17 Pour la structuration et le stockage dans un système d'information, nous avons choisi d'avoir recours à une base de données relationnelle pour recomposer la complexité, la multiscalarité et l'hétérogénéité structurelle, spatio-temporelle et narrative de Nedjma que nous avons décrite jusque-là - complexité que le langage générique XML (eXtensible Markup Language), très courant dans le traitement numérique des textes, garantissant simplicité et interopérabilité, ne pouvait pleinement respecter (voir figure 6 et figure 7). 
Figure 6. Superposition et enchaînement des unités narratives et textuelles dans l'extrait du chapitre III de Nedjma

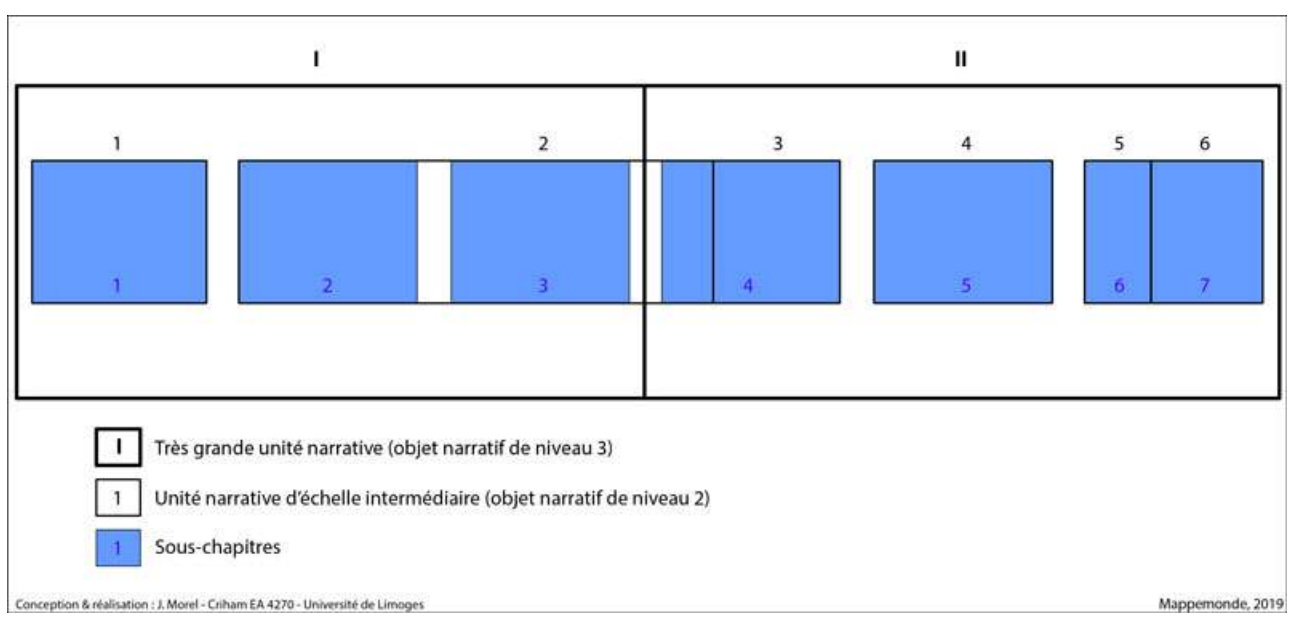

Conception \& réalisation : J. Morel- CRIHAM, Université de Limoges

Figure 7. Traduction en XML de la structure formelle et spatiale du récit de Nedjma

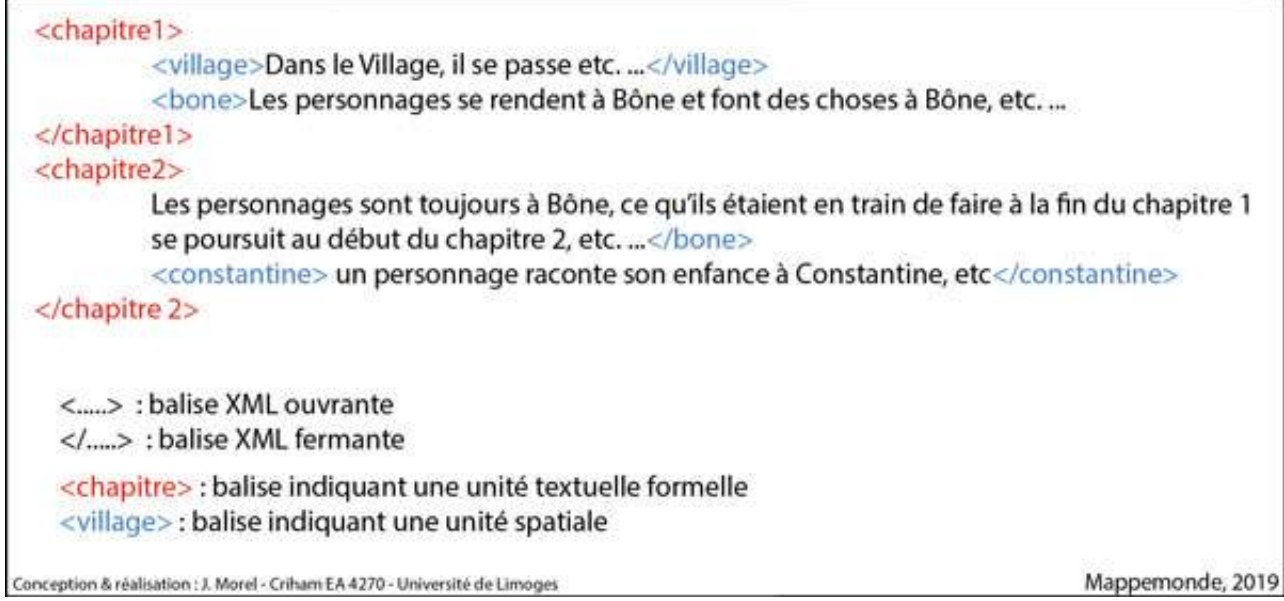

Cette traduction est impossible puisqu'un tel découpage provoque un chevauchement des balises XML (erreur de syntaxe, les éléments XML sont nécessairement imbriqués)

Conception \& réalisation: J. Morel- CRIHAM, Université de Limoges

En effet, le métalangage XML n'admet pas le chevauchement des balises découpant le texte, il est strictement hiérarchique, alors qu'une base de données relationnelle connecte des objets hétérogènes de manière horizontale autant que verticale et permet des interrogations multidimentionnelles. Cette dernière permet en outre de stocker des informations purement géographiques, les géométries, ce qui est évidemment fondamental dans le cas de notre étude sur les relations entre narration et géographie. 
Figure 8. Schéma simplifié de la structure de base de données relationnelle du Cycle de Nedjma

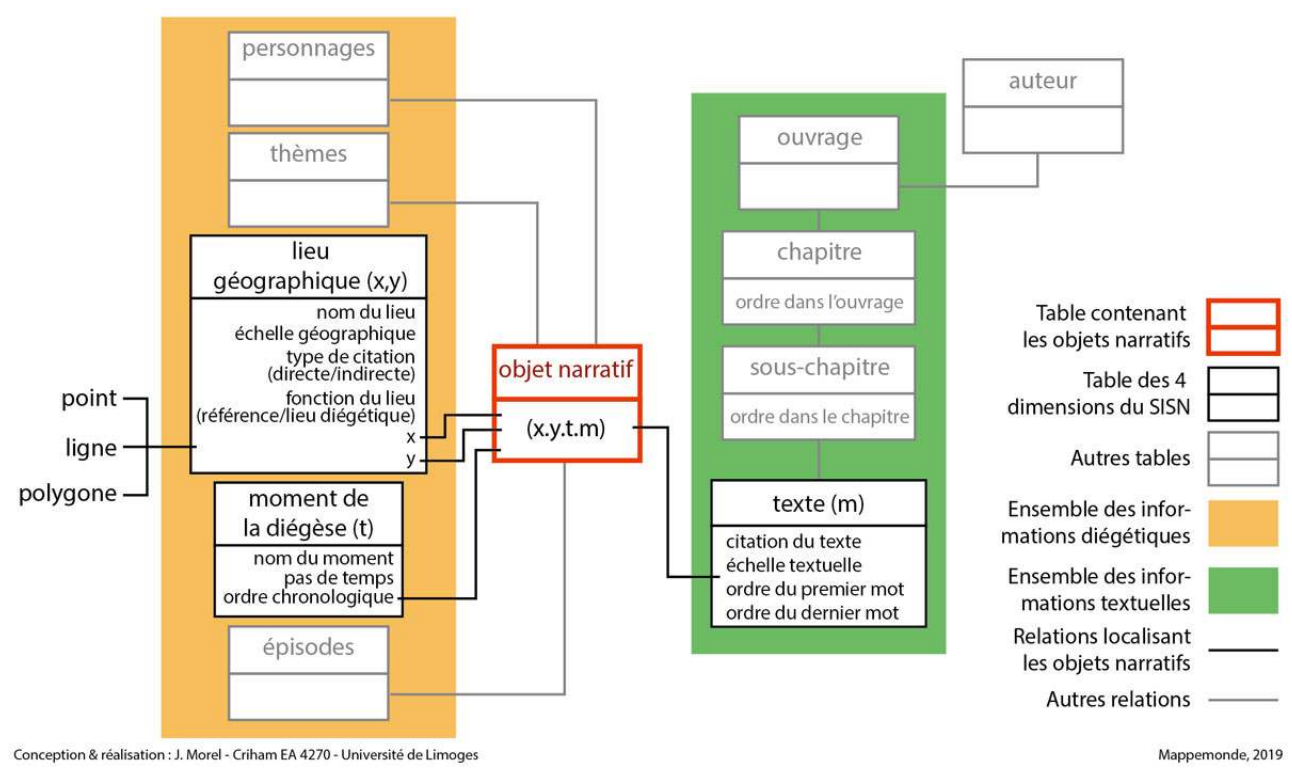

Conception \& réalisation : J. Morel- CRIHAM, Université de Limoges

19 La base de données relationnelle qui regroupe les informations spatio-narratives selon les principes que nous avons décrits jusque-là est construite en trois parties (voir la figure 8 ). Une première partie stocke les informations se rapportant à la diégèse (sur fond jaune). Elle regroupe des tables référençant les personnages, les thèmes, les épisodes diégétiques, les moments de l'histoire et les lieux. Ces cinq catégories sont considérées comme résumant schématiquement le contenu de la diégèse, l'objectif étant de mettre en lumière les itérations diégétiques au sein du roman. Une deuxième partie de la base de données référence les informations liées à la narration (sur fond vert). Cette partie rassemble des tables qui stockent des informations purement textuelles: les unités définies concrètement dans le texte - les ouvrages, les chapitres, les sous-chapitres -, ainsi que le texte lui-même stocké en intégralité dans la table "texte ». Entre ces deux pôles se trouve la partie centrale de la base de données stockant les objets narratifs (en rouge). Tel que nous l'avons expliqué ci-dessus, ceux-ci sont définis dans ce système comme des citations situées dans la matérialité du texte d'une part (dans les sous-chapitres et chapitres) et dans la diégèse d'autre part (par leur liaison avec les tables référençant les moments et les lieux). Cette base de données donne une importance particulière aux informations géographiques et à leurs différentes caractéristiques et fonctions que nous avons soulignées dans cet article. Elle intègre une table «lieu géographique », qui centralise et synthétise toutes les indications géographiques citées et tous les espaces accueillant des épisodes narratifs dans Nedjma, en les considérant indifféremment comme des points cartographiques, ceci afin de pouvoir mettre en commun sous une même forme tous les espaces intervenants dans le récit. Trois tables complètent cette table «lieu», en reprenant respectivement les objets géographiques contenus dans cette dernière, mais en les considérant cette fois selon la géométrie que leur confère l'écriture, ce afin de rendre compte plus finement de la géographie littéraire construite. Les trois implantations fondamentales de la cartographie - le point, la ligne et la surface - se retrouvent en effet dans l'écriture géographique de l'auteur, dans la manière littéraire dont Kateb Yacine traite les différents espaces (Morel, 2014). 


\section{Conclusion}

La lecture des cartes et graphiques réalisés à partir d'une modélisation du système spationarratif de Nedjma de Kateb Yacine dont nous avons développé les principes dans cet article nous apprend beaucoup sur la construction spatiale du récit et, inversement, sur la construction narrative de l'espace. Nous pouvons par exemple lire sur cette proposition de visualisation graphique ci-dessous la confirmation de la création, par le récit, d'ensembles géographiques. Ressortent des espaces géographiquement et narrativement cohérents : les étapes du voyage vers la Mecque (voir 1 sur la figure 9) et le Nadhor (2). On observe également qu'au début et à la fin du récit un rapprochement est opéré entre le village chantier et Bône (3) et que les villes de X., Sétif et Guelma - villes de l'enfance sont systématiquement regroupées (4). Alors que le récit est encadré par ces ensembles géo-narratifs, son centre est marqué par le couple Constantine-Bône (5). Les deux villes transcendent les époques historiques et diégétiques, mais on voit que l'une et l'autre bénéficient d'un statut différent: les récits situés à Constantine (6) couvrent une temporalité plus ample que ceux situés à Bône. Constantine provoque l'achronie narrative - ce qui concrétise narrativement le concept de ville palimpseste -, alors que Bône concentre le récit dans le temps (autour du nœud diégétique que constitue l'amour des protagonistes pour Nedjma).

Figure 9. Structures narratives et géographiques dans Nedjma de Kateb Yacine

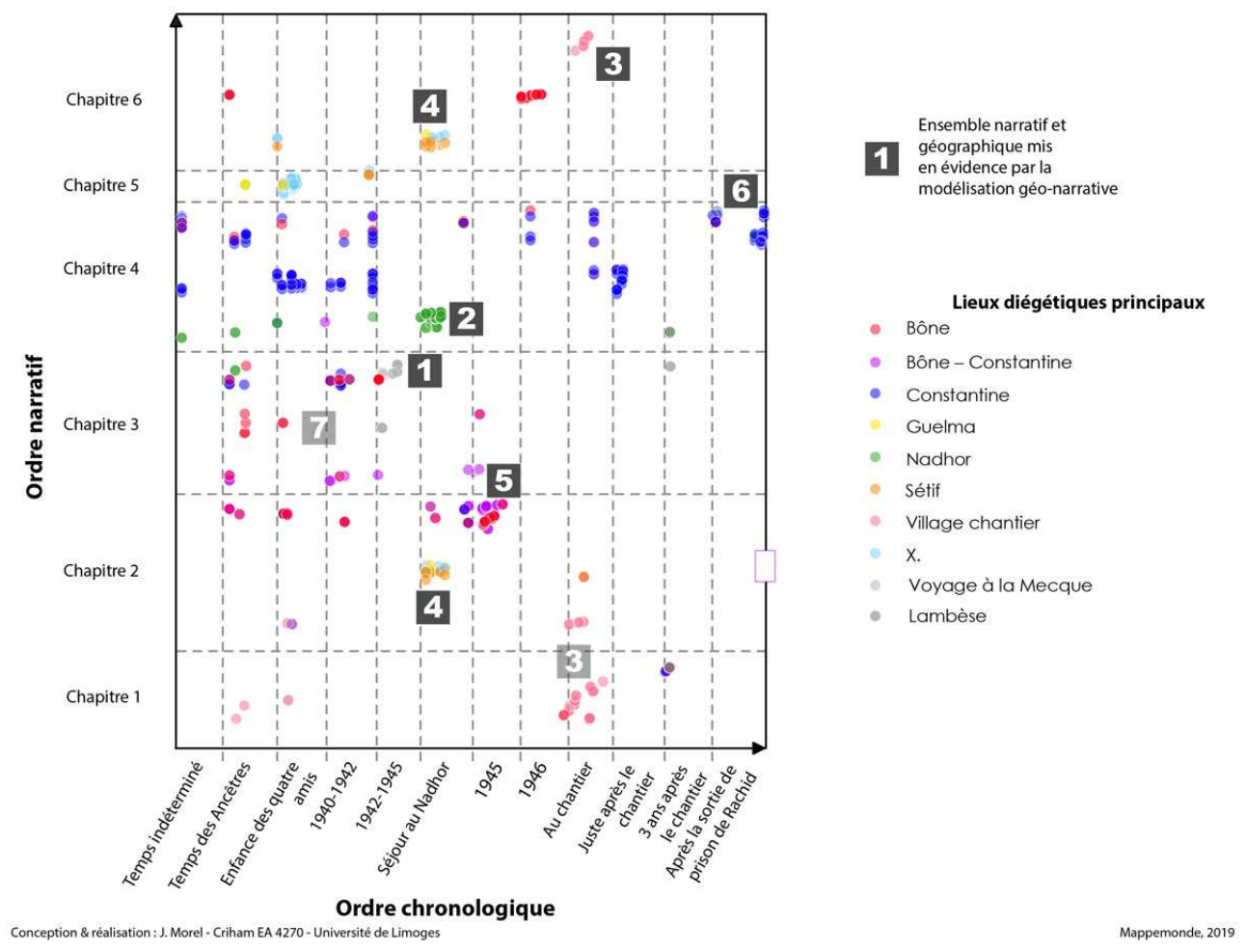

Conception \& réalisation : J. Morel- CRIHAM, Université de Limoges

Au-delà de ces enseignements spécifiques à l'œuvre de Kateb Yacine, nous pouvons tirer trois conclusions générales concernant l'interdisciplinarité de la méthodologie mise en œuvre. Premièrement, l'influence de la "littérarité » d'un texte sur l'espace peut 
s'observer à différentes échelles - ici à l'échelle de la structure du récit et des enchaînements narratifs -, et non seulement à l'échelle fine des figures poétiques et de la symbolique - endroit où se fixe souvent l'attention des études géo-littéraires. Deuxièmement, la démarche de la modélisation - comme la démarche des humanités numériques en général - oblige à appliquer un traitement systématique et exhaustif, et à adopter une vision d'ensemble, ce qui permet de modifier le point de vue sur le texte, de se libérer de la linéarité du discours critique - parfois impropre à synthétiser la complexité - et donc de rendre visibles certains processus invisibles autrement. Troisièmement, cette étude explore voire éprouve le sens propre que l'on peut donner à la notion d'espace littéraire. Elle montre que le récit, dans sa dimension la plus formelle, participe à construire un système que seules des modélisations multidimentionnelles et multiscalaires peuvent approcher. Cette méthodologie s'offre ainsi comme une proposition de modèle de formalisation de système narratif complexe, reproductible pour explorer le rôle de l'espace dans les récits complexes ou « post-modernes ».

\section{BIBLIOGRAPHIE}

ARNAUD J. (1986). La Littérature maghrébine de langue française. II : le cas de Kateb Yacine. Paris :

Éditions Publisud, coll. «Espaces méditerranéens », 741 p.

BARTHES R. (1966). «Introduction à l'analyse structurale des récits ». Communications, vol. 8, nº 1, p. 1-27.

BERQUE A. (1996). Être humains sur la Terre, principes d'éthique de l'écoumène. Paris : Gallimard, 216 p. ISBN 9782070745494.

BUTOR M. (1992). Essais sur le roman. Paris : Gallimard, coll. « Tell ».

BLANCHOT M. (1955). L'espace littéraire. Paris : Gallimard, coll. « Folio essais ».

BONN C. (2000). Kateb Yacine ; Nedjma. Paris : L'Harmattan.

DI MEO G. (2001[1998]). Géographie sociale et territoires. Paris : Nathan université, coll. « Fac. Géographie ».

GARNIER X., ZOBERMAN P., dir. (2006). Qu'est-ce qu'un espace littéraire ? Saint-Denis : PUV, coll. «L'imaginaire du texte ».

GENETTE G. (1966). Figures I. Paris : Éditions du Seuil, coll. « Tel Quel ».

GENETTE G. $(2007$ [1972, 1983]). Discours du récit. Paris : Éditions du Seuil, coll. « Point essais », 448 p. EAN 9782757805381

GenetTe G. (1982). Palimpseste. Paris : Éditions du Seuil, coll. « Poétique », 480 p.

EAN 9782020061162

GUERE H. (1990). « Le sens du sens. Essai sur l'espace littéraire ». In FRÖHLICHER P., GÜNTERT G. ET THÜRLEMANN F. Espaces du texte. Recueil d'hommages pour Jacques Geninasca, Éditions de la

Braconnière, coll. « Langages », 421 p. 
GONTARD M. (1985). Nedjma de Kateb Yacine, essai sur la structure formelle du roman. Paris :

L'Harmattan, $124 \mathrm{p}$.

HOover D. L. (2007). "Quantitative Analysis and Literary Studies”. In Companion to Digital Literary

Studies, mis en ligne en 2007, consulté le 15 juillet 2016. En ligne : http://

digitalhumanities.org:3030/companion/view?

docId=blackwell/9781405148641/9781405148641.xml\&chunk.id=ss1-6-9\&toc.depth=1\&toc.id=ss1-6-9\&brand=9781405148641_brand

KATEB Y. (1956). Nedjma. Paris : Éditions du Seuil.

KATEB Y. (1959). Le Cercle des représailles. Paris : Éditions du Seuil. Composé des pièces : Le Cadavre

encerclé, La Poudre d'Intelligence, Les Ancêtres redoublent de férocité, Le Vautour.

KATEB Y. (1966). Le Polygone étoilé. Paris : Éditions du Seuil.

KATEB Y. (1986). L'Euvre en Fragments. Inédits littéraires et textes rassemblés et présentés par

Jacqueline Arnaud. Paris : Éditions Sindbad, coll. « La bibliothèque arabe ».

KATEB Y. (1994). Le Poète comme un boxeur : entretiens 1958-1989. Textes réunis et présentés par Gilles Carpentier avec le concours de l'IMEC, Paris : Éditions du Seuil, 184 p. ISBN 9782020221931

KHADDA N. (2002). « Kateb : écrivain d'avant-garde, engagement politique et construction

d'identité ». In GIRAULT J., LECHERBONNIER B. (dir.), Kateb Yacine un intellectuel dans la révolution

algérienne, Revue Itinéraires et Contacts de cultures, n³2, Paris : L'Harmattan, 200 p. ISBN :

2-7475-3097-3

LefEBVRe H. (2000 [1974]). La Production de l'espace. Paris : Éditions Anthropos, coll.

«Ethnosociologie », 485 p.

LÉVY J., LUSSAULT M. (2003). « Espace ». In LÉVY J., LUSSAULT M. (dir.), Le Dictionnaire de la géographie et de l'espace des sociétés. Paris : Éditions Belin.

MOREL J. (2014). « Kateb Yacine : l'écrivain-cartographe ». In CHIKHI B., DOUAIRE-BANNY A. (dir.), Kateb Yacine. Au cœur d'une histoire polygonale, Rennes : Presses universitaires de Rennes.

ISBN 978-2-7535-3275-5

MOREL J., ROUIAÏ N. (2016). « Cartographier les films. L'exemple de la cartographie de Hong-Kong au cinéma ». Mappemonde, $\mathrm{n}^{\circ}$ 118. En ligne $:$ http://mappemonde.mgm.fr/118as3/

PLUMEJEAUD C., CRISTOFOLI P., MOTTE C. (2015). « De l'étude des nomenclatures territoriales à la modélisation des dynamiques des territoires administratifs en France ». Revue internationale de Géomatique, vol. 25, n 3.

RODIER X., SALIGNY L. (2010). « Modélisation des objets historiques selon la fonction, l'espace et le temps pour l'étude des dynamiques urbaines dans la longue durée ». Cybergeo : European Journal of Geography, Systèmes, Modélisation, Géostatistiques, document 502, mis en ligne le 17 juin 2010, consulté le 26 mars 2018. En ligne http://journals.openedition.org/cybergeo/23175; DOI :

$10.4000 /$ cybergeo. 23175

\section{NOTES}

1. «Bône » est le nom colonial que porte jusqu'à l'indépendance de l'Algérie la ville aujourd'hui nommée Annaba, située sur la côte est de l'Algérie. Nous conservons les toponymes utilisés par Kateb Yacine pour désigner les lieux dont il est question dans son œuvre. 
2. Par exemple le sous-chapitre 9 du chapitre VI fait cinq mots: «Les étrangers seront-ils congédiés? ", Nedjma, op.cit, p. 241.

3. Nous pensons notamment aux formules (mots ou phrases) martelées dans les ouvrages de Kateb Yacine, comme la phrase « Chaque fois, les plans sont bouleversés » dans Le Polygone étoilé ou les phrases « Invivable consomption du zénith ! prémices de fraîcheur... » dans Nedjma.

4. Un seul lieu pourrait avoir un statut imaginaire dans le roman Nedjma, la région mythique du Nadhor. Néanmoins, la dimension imaginaire du Nadhor tient à ce qui s'y passe non à sa localisation : cette région renvoie à une région réelle d'Algérie située autour du Mont Nadhor à l'est de Constantine.

\section{RÉSUMÉS}

L'œuvre littéraire de Kateb Yacine peut-être doublement appréhendée par l'espace : parce qu'elle représente l'espace algérien et met en scène des problématiques géopolitiques réelles et parce qu'elle construit un système narratif complexe, conceptualisé et organisé comme un espace géométrique. Nous proposons d'étudier la co-construction et l'influence mutuelle de ces deux domaines spatiaux grâce à une méthode de modélisation spatiale du récit. Le principe de cette modélisation géo-narrative s'inspire des systèmes d'information géographique et spatiotemporelle - parce que le récit est le résultat d'une manipulation du temps et de l'espace par la narration - pour décrire, stocker et explorer par un même système d'information la structure du récit et la géographie de l'œuvre.

Kateb Yacine's literary works can be read through a spatial lens at two different levels: First, they represent Algerian space and raises geopolitical issues. Second, their narrative structure is complex and spatially conceptualized and organized, as if they took place in a geometric space. This article describes a method built to modelize the co-construction and the mutual influence of both these spaces. This geo-narrative modelization is based on the principles of geographical and spatio-temporal information systems - since a narrative system can be described as a manipulation of space and time.

La obra literaria de Kateb Yacine se puede entender desde dos planteamientos espaciales: representa el territorio argelino e introduce su problemática geopolítica, y construye un sistema narrativo complejo conceptualizado y organizado como si fuera un espacio geométrico. Este artículo propone estudiar la co-construcción e interdependencia de estos dos planteamientos mediante la modelización espacial de su obra literaria. Esta conceptualización geo-narrativa se fundamenta en los sistemas de información geográficos y en un modelo espacio-temporal partiendo de la premisa por la que una narración es el resultado de la manipulación del espacio y del tiempo del propio relato- para describir, organizar y explorar mediante el sistema de información la estructura y geografía de la obra. 
INDEX

Mots-clés : espace littéraire, système d'information géo-narratif, Kateb (Yacine), récit Thèmes : La mise en carte des récits

Palabras claves : espacio literario, sistema de información geo-narrativo, Kateb (Yacine), narración

Keywords : literary space, geo-narrative information system, Kateb (Yacine), story

\section{AUTEUR}

JULIETTE MOREL

CRIHAM, Université de Limoges 